\title{
SIMULTANEOUS EXTENSION OF PARTIAL ENDOMORPHISMS OF GROUPS
}

\author{
by C. G. CHEHATA
}

(Received 5th June, 1953)

\section{Introduction.}

Let $\mu$ be a homomorphic mapping of some subgroup $A$ of the group $G$ onto a subgroup $\dot{B}$ (not necessarily distinct from $A$ ) of $G$; then we call $\mu$ a partial endomorphism of $G$. If $A$ coincides with $G$, that is, if the homomorphism is defined on the whole of $G$, we speak of a total endomorphism; this is what is usually called an endomorphism of $G$. A partial (or total) endomorphism $\mu^{*}$ extends or continues a partial endomorphism $\mu$ if the domain of $\mu^{*}$ contains the domain of $\mu$, that is, $\mu^{*}$ is defined for (at least) all those elements for which $\mu$ is defined, and moreover $\mu^{*}$ coincides with $\mu$ where $\mu$ is defined.

In this paper, I deal with the simultaneous extension of several partial endomorphisms of $G$ to total endomorphisms of a supergroup of $G$. The starting point is a paper by B. $\mathrm{H}$. Neumann and Hanna Neumann (2) in which necessary and sufficient conditions are given for this extension to be possible for a single partial endomorphism. Here these conditions are generalized to apply first to two partial endomorphisms, and then, using transfinite induction, to any well-ordered set of partial endomorphisms. The conditions here obtained are again necessary and sufficient. A number of special consequences are derived, in analogy to results of the paper by B. H. Neumann and Hanna Neumann already referred to ; a typical corollary is the following :

Any number of partial endomorphisms of an abelian group can be extended to total endomorphisms of an abelian supergroup.

The principal tool throughout is the free product with one amalgamated subgroup (in the case of abelian groups the direct product with an amalgamated subgroup is used instead).

I wish to express my indebtedness to Dr. B. H. Neumann for his generous advice and help during the work.

\section{$\S 1$. Necessary Conditions.}

Let $\mu$ and $\nu$ be two partial endomorphisms of $G$ mapping $A$ onto $B$ and $C$ onto $D$ respectively ; $A, B, C$ and $D$ being subgroups of $G$. Now to derive the necessary conditions for $\mu$ and $\nu$ to be totally extendable, we assume that the extension is already established, that is to say, take the group $G^{*} \supseteq G$ and its total endomorphisms $\mu^{*}$ and $\nu^{*}$ which extend $\mu$ and $\nu$ respectively as given.

Denote by $\Omega^{*}$ the semigroup generated by $\mu^{*}$ and $\nu^{*}$. Then any $\omega^{*} \epsilon \Omega^{*}$ is an endomorphism of $G^{*}$, denote the kernel of $\omega^{*}$ by $K\left(\omega^{*}\right)$.

If we denote the kernel of $\mu$ by $K$, then the canonic mapping of $G^{*}$ onto $G^{*} / K\left(\mu^{*}\right)$ must induce the canonic mapping of $A$ onto $A / K$; but the former induces the canonic mapping of $A$ onto $A /\left(K\left(\mu^{*}\right) \sim A\right)$, thus

$$
K=K\left(\mu^{*}\right) \cap A=\text { kernel of } \mu
$$

In a similar manner one proves that

$$
K\left(\nu^{*}\right) \cap C=\text { kernel of } \nu .
$$

Let $\Omega$ be the free semigroup with two generators, which we also denote ambiguously, 
but conveniently, by $\mu$ and $\nu$. Then to every element in $\Omega$, that is to every word $\omega=\omega(\mu, \nu)$, there corresponds an element $\omega^{*}=\omega\left(\mu^{*}, \nu^{*}\right)$ of $\Omega^{*}$. Define

$$
L_{\omega}=K\left(\omega^{*}\right) \cap G \text {, }
$$

for every word $\omega \epsilon \Omega$ and the corresponding element $\omega^{*} \epsilon \Omega^{*}$.

Then equations (1.1) and (1.2) will become

$$
\begin{aligned}
& L_{\mu} \cap A \text { is the kernel of } \mu, \\
& L_{\nu} \cap C \text { is the kernel of } \nu .
\end{aligned}
$$

For any $\omega^{*} \epsilon \Omega^{*}$ we have

$$
K\left(\mu^{*} \omega^{*}\right)=K\left(\omega^{*}\right) \mu^{*-1}
$$

for if $x \in K\left(\mu^{*} \omega^{*}\right)$, then $x \mu^{*} \omega^{*}=1$ or

thus

$$
\begin{gathered}
x \mu^{*} \in K\left(\omega^{*}\right), \\
x \in K\left(\omega^{*}\right) \mu^{*-1}, \\
K\left(\mu^{*} \omega^{*}\right) \subseteq K\left(\omega^{*}\right) \mu^{*-1} ;
\end{gathered}
$$

if, on the other hand, $x \in K\left(\omega^{*}\right) \mu^{*-1}$, then

thus

$$
\begin{aligned}
& x \mu^{* *} \omega^{*}=1, \\
& x \in K\left(\mu^{*} \omega^{*}\right),
\end{aligned}
$$

this proves relation (1.4).

Similarly we have

$$
K\left(\nu^{*} \omega^{*}\right)=K\left(\omega^{*}\right) \nu^{*-1},
$$

for any $\omega^{*} \epsilon \Omega^{*}$.

Now define

and

$$
\begin{aligned}
P\left(\mu^{*} \omega^{*}\right) & =K\left(\mu^{*} \omega^{*}\right) \cap A \\
& =L_{\mu \omega} \cap A, \text { by }(1.3), \\
Q\left(\nu^{*} \omega^{*}\right) & =K\left(\nu^{*} \omega^{*}\right) \cap C \\
& =L_{\nu \omega} \cap C
\end{aligned}
$$

for any $\omega^{*} \epsilon \Omega^{*}$.

Taking $\omega^{*}$ arbitrary and applying $\mu$ to $P\left(\mu^{*} \omega^{*}\right)$, we get

$$
\begin{aligned}
P\left(\mu^{*} \omega^{*}\right) \mu & =P\left(\mu^{*} \omega^{*}\right) \mu^{*}, \text { since } \mu^{*} \text { extends } \mu, \\
& =\left(K\left(\mu^{*} \omega^{*}\right) \cap A\right) \mu^{*} \\
& \subseteq K\left(\mu^{*} \omega^{*}\right) \mu^{*} \cap A \mu^{*} \\
& \subseteq K\left(\omega^{*}\right) \cap B,
\end{aligned}
$$

since (1.4) implies $K\left(\mu^{*} \omega^{*}\right) \mu^{*} \subseteq K\left(\omega^{*}\right)$. Thus

$$
P\left(\mu^{*} \omega^{*}\right) \mu \subseteq L_{\omega} \cap B .
$$

If, on the other hand, $b \in L_{\omega} \cap B$, then there exists an element $a \in A$ such that $a \mu=b$; thus

and

$$
\begin{gathered}
a \mu^{*}=a \mu \in L_{\omega} \subseteq K\left(\omega^{*}\right), \\
a \in K\left(\omega^{*}\right) \mu^{*-1}=K\left(\mu^{*} \omega^{*}\right) \quad \text { by }(1.4), \\
a \in K\left(\mu^{*} \omega^{*}\right) \cap A=P\left(\mu^{*} \omega^{*}\right), \\
\quad b \in P\left(\mu^{*} \omega^{*}\right) \mu \\
\quad L_{\omega} \cap B \subseteq P\left(\mu^{*} \omega^{*}\right) \mu ;
\end{gathered}
$$

hence we have finally,

$$
P\left(\mu^{*} \omega^{*}\right) \mu=\left(L_{\mu \omega} \cap A\right) \mu=L_{\omega} \cap B
$$


Similarly we can prove that

for any $\omega^{*} \epsilon \Omega^{*}$.

$$
Q\left(\nu^{*} \omega^{*}\right) \nu=\left(L_{\nu \omega} \cap C\right) \nu=L_{\omega} \cap D
$$

Moreover, we note that for any $\omega^{*}, \omega_{1}^{*} \in \Omega^{*}, a \in K\left(\omega^{*}\right)$ implies $a \omega^{*}=1$ and $a \omega^{*} \omega_{1}^{*}=1$; that is to say $a \in K\left(\omega^{*} \omega_{1}^{*}\right)$; thus

$$
K\left(\omega^{*}\right) \subseteq K\left(\omega^{*} \omega_{1}^{*}\right)
$$

which in turn gives, because of (1.3) :

$$
L_{\omega} \subseteq L_{\omega \omega_{1}}
$$

Finally, the kernels $K\left(\omega^{*}\right)$ for all $\omega^{*} \epsilon \Omega^{*}$ are normal subgroups of $G^{*}$; hence $L_{\omega}$ for all $\omega \epsilon \Omega$ are normal subgroups of $G$. If we pick out those conditions which involve the given groups $G, A, B, C, D$ and the given partial endomorphisms $\mu$ and $\nu$ only, we obtain the following

\section{Theorem 1.}

If we denote the semigroup freely generated by $\mu$ and $\nu$ by $\Omega$, then the following conditions are necessary for $\mu$ and $\nu$ to be simultaneously extendable to total endomorphisms of one and the same group : For each $\omega \in \Omega$ there exists a normal subgroup $L_{\omega}$ of $G$ such that :

$$
\begin{aligned}
& L_{\omega} \subseteq L_{\omega \omega_{1}} \text { for any } \omega, \omega_{1} \epsilon \Omega ; \\
& L_{\mu} \cap A \text { is the kernel of } \mu, \ldots . \\
& L_{\nu} \cap C \text { is the kernel of } \nu ; \ldots \\
& \left(L_{\mu \omega} \cap A\right) \mu=L_{\omega} \cap B, \ldots \ldots \ldots . \\
& \left(L_{\nu \omega} \cap C\right) \nu=L_{\omega} \cap D ; \ldots \ldots \ldots
\end{aligned}
$$

for any $\omega \in \Omega$.

\section{§2. Sufficiency of the Conditions.}

Before proving that the conditions (1.5)-(1.9) of Theorem 1 are also sufficient, we mention here three lemmas proved by B. H. Neumann and Hanna Neumann $(2, \S 4)$ :

Lemma 1 :

Let $Q \supseteq S \supseteq U$ and $R \supseteq T \supseteq V$ be groups and $\theta$ a homomorphic mapping of $Q$ onto $R$, mapping $S$ but no bigger group onto $T$, and $U$ but no bigger group onto $V$ (that is to say, $U$ contains the kernel of $\theta$ ). Then

is equivalent to

$$
\begin{aligned}
& U^{Q} \cap S=U \\
& V^{R} \cap T=V,
\end{aligned}
$$

where $U^{Q}$ stands for the normal closure of $U$ in $Q$.

Lemma 2 :

Let $P$ be the free product of two groups $Q, R$ with an amalgamated subgroup $S$. Let $U$ be a normal subgroup of $Q$. Then the two equations

are equivalent and imply

$$
\begin{gathered}
U^{P} \cap Q=U, \\
(U \cap S)^{R} \cap S=U \cap S
\end{gathered}
$$

Lemma 3 :

$$
U^{P} \cap R=(U \cap S)^{R}
$$

Let $P, Q, R, S, U$ be as in Lemma 2, and let $V$ be a normal subgroup of $R$. Assume

$$
\begin{aligned}
& U^{P} \cap Q=U, \\
& V^{P} \cap R=V, \\
& U \cap S=V \cap S .
\end{aligned}
$$


Putting $W=U \cup V$, then

$$
W^{P}{ }_{n} Q=U, \quad W^{P} \cap R=V .
$$

Now assume that the necessary conditions (1.5)-(1.9) are satisfied. Using the notation of $\S 1$, let

then $H$ contains a subgroup

$$
H=G \mid L_{\mu}
$$

$$
B^{\prime}=A \cup L_{\mu} / L_{\mu} \cong A / A \cap L_{\mu} \cong B,
$$

since $A \cap L_{\mu}$ is the kernel of $\mu$. An jsomorphism between $B^{\prime}$ and $B$ is defined by letting $a L_{\mu} \in B^{\prime}$ correspond to $a \mu \in B$ where $a \in A$.

We then define $G_{1}$ as the free product of $G$ and $H$, amalgamating $B$ and $B^{\prime}$ according to this isomorphism :

$$
G_{1}=\left\{G * H ; B=A \cup L_{\mu} / L_{\mu}\right\}
$$

Denote by $\mu_{1}$ the canonic mapping of $G$ onto $H$. This maps $A$ onto $B$ according to $\mu$, that is to say, it continues $\mu$ to $G$.

We shall prove that in $G_{1}$ we can define a set of normal subgroups $M_{\omega}$ such that the relations (1.5)-(1.9) of Theorem 1 will be satisfied with $G_{1} ; G, H, C, D ; \mu_{1}, \nu ; M_{\omega}$ taking the place of $G ; A, B, C, D ; \mu, \nu ; L_{\omega}$. Before defining $M_{\omega}$, we prove the following:

\section{Lemma 4 :}

The following relations hold for any $\omega \in \Omega$ :

$$
\begin{aligned}
L_{\omega}^{G_{1}} \cap G & =L_{\omega}, \ldots . \\
\left(L_{\mu \omega} \mu_{1}\right)^{G_{1}} \cap H & =L_{\mu \omega} \mu_{1}, \\
L_{\mu \omega} \mu_{1} \cap B & =L_{\omega} \cap B .
\end{aligned}
$$

Proof.

Since $L_{\mu \omega}$ is normal in $G$, then

but since

$$
\left(L_{\mu \omega} \cap\left(A \cup L_{\mu}\right)\right)^{G} \subseteq L_{\mu \omega},
$$

$$
\left(L_{\mu \omega} \cap(A \cup L)\right)^{G} \cap\left(A \cup L_{\mu}\right) \text { 드 } L_{\mu \omega} \cap\left(A \cup L_{\mu}\right)
$$

and

$$
L_{\mu \omega} \cap\left(A \cup L_{\mu}\right) \subseteq\left(L_{\mu \omega} \cap\left(A \cup L_{\mu}\right)\right)^{G}
$$

then

$$
\begin{aligned}
& L_{\mu \omega}\left(A \cup L_{\mu}\right) \subseteq A \cup L_{\mu}, \\
& L_{\mu \omega} \cap\left(A \cup L_{\mu}\right) \subseteq\left(L_{\mu \omega} \cap\left(A \cup L_{\mu}\right)\right)^{G} \cap\left(A \cup L_{\mu}\right) .
\end{aligned}
$$

(2.4) and (2.5) together give

$$
\left(L_{\mu \omega} \cap\left(A \cup L_{\mu}\right)\right)^{G} \cap\left(A \cup L_{\mu}\right)=L_{\mu \omega} \cap\left(A \cup L_{\mu}\right)
$$

Now $\mu_{1}$ is a homomorphism of $G$ onto $H$ which maps $A \cup L_{\mu}$ onto $B$. Since $A \cup L_{\mu}$ contains the kernel $L_{\mu}$ of $\mu_{1}$ then it is the biggest group mapped by $\mu_{1}$ onto $B$. We also have

and since $L_{\mu} \subseteq L_{\mu \omega}$, by (1.5), implies

$$
\begin{aligned}
\left(L_{\mu \omega} \cap\left(A \cup L_{\mu}\right)\right) \mu_{1} & =\left(L_{\mu \omega} \cap A\right) \mu_{1} \\
& =\left(L_{\mu \omega} \cap A\right) \mu, \text { since } \mu_{1} \text { extends } \mu, \\
& =L_{\omega} \cap B, \text { by }(1.8) ;
\end{aligned}
$$

$$
L_{\mu} \subseteq L_{\mu \omega} \cap\left(A \cup L_{\mu}\right)
$$

then $\mu_{1}$ maps $L_{\mu \omega} \cap\left(A \cup L_{\mu}\right)$, but no bigger group, onto $L_{\omega} \cap B$. Since

and

$$
G \supseteq A \cup L_{\mu} \supseteq L_{\mu \omega} \cap\left(A \cup L_{\mu}\right)
$$$$
H \equiv B \equiv L_{\omega} \cap B \text {, }
$$

then applying Lemma 1 with $G, A \cup L_{\mu}, L_{\mu \omega} \cap\left(A \cup L_{\mu}\right) ; H, B, L_{\omega} \cap B ; \mu_{1}$ taking the place of $Q, S, U ; R, T, V ; \theta$ respectively, we find that (2.6) is equivalent to :

$$
\left(L_{\omega} \cap B\right)^{H} \cap B=L_{\omega} \cap B .
$$


Applying Lemma 2, with $G_{1}, G, H, B, L_{\omega}$ in the place of $P, Q, R, S, U$ respectively, then (2.7) is equivalent to

$$
L_{\omega}^{G_{1}} \cap G=L_{\omega}
$$

proving (2.1).

Consider the left-hand side of (2.3) which is

$$
L_{\mu \omega} \mu_{1}=L_{\mu \omega} \mu_{1} \cap A \mu_{1} \equiv\left(L_{\mu \omega} \cap A\right) \mu_{1} \text {. }
$$

If $b \in L_{\mu \omega} \mu_{1} \cap A \mu_{1}$, then there exist elements $l_{\mu \omega} \in L_{\mu \omega}$ and $a \epsilon A$ such that

$$
b=l_{\mu \omega} \mu_{1}=a \mu_{1} \text {; }
$$

thus $l_{\mu \omega}^{-1} a \in L_{\mu}$, that is to say,

$$
a=l_{\mu \omega} l_{\mu} \text { for some } l_{\mu} \in L_{\mu} .
$$

But $L_{\mu} \subseteq L_{\mu \omega}$, by (1.5); thus $a \in L_{\mu \omega}$ and

$$
b \in\left(L_{\mu \omega} \cap A\right) \mu_{1} \text {. }
$$

Hence

$$
L_{\mu \omega} \mu_{1} \cap A \mu_{1} \subseteq\left(L_{\mu \omega} \cap A\right) \mu_{1} \text {. }
$$

(2.8) together with (2.9) gives

$$
\left(L_{\mu \omega} \cap A\right) \mu_{1}=L_{\mu \omega} \mu_{1} \cap B .
$$

But

$$
\begin{aligned}
\left(L_{\mu \omega} \cap A\right) \mu_{1} & =\left(L_{\mu \omega} \cap A\right) \mu \\
& =L_{\omega} \cap B, \quad \text { by (1.8). }
\end{aligned}
$$

Thus (2.10) gives

$$
L_{\mu \omega} \mu_{1} \cap B=L_{\omega} \cap B
$$

proving (2.3)

Finally, since $L_{\omega}$ is normal in $G$, then

$$
\left(L_{\omega} \cap B\right)^{Q} \subseteq L_{\omega}
$$

thus

$$
\left(L_{\omega} \cap B\right)^{G} \cap B \subseteq L_{\omega} \cap B \text {; }
$$

but also, obviously,

$$
\left(L_{\omega} \cap B\right)^{\alpha} \cap B \supseteq L_{\omega} \cap B \text {, }
$$

and thus

$$
\left(L_{\omega} \cap B\right)^{\alpha} \cap B=L_{\omega} \cap B .
$$

Using (2.3) to replace $L_{\omega} \cap B$ by $L_{\mu \omega} \mu_{1} \cap B$, we get

$$
\left(L_{\mu \omega} \mu_{1} \cap B\right)^{G} \cap B=L_{\mu \omega} \mu_{1} \cap B .
$$

To this we apply Lemma 2 with $G_{1}, H, G, B, L_{\mu \omega} \mu_{1}$ taking the place of $P, Q, R, S, U$ respectively. We then get

$$
\left(L_{\mu \omega} \mu_{1}\right)^{G} H=L_{\mu \omega} \mu_{1},
$$

proving (2.2). This completes the proof of Lemma 4.

Now define

$$
M_{\omega}=\left(L_{\mu \omega} \mu_{1} \cup L_{\omega}\right)^{G_{1}},
$$

for every $\omega \in \Omega$.

Replacing $P, Q, R, S, U, V, W^{P}$ of Lemma 3 by $G_{1}, G, H, B, L_{\omega}, L_{\mu \omega} \mu_{1}, M_{\omega}$ respectively, the relations (2.1)-(2.3) of Lemma 4 translate into the conditions of Lemma 3. Thus we can apply Lemma 3 to get the following two equations

$$
\begin{aligned}
& M_{\omega} \cap G=L_{\omega}, . \\
& M_{\omega} \cap H=L_{\mu \omega} \mu_{1} .
\end{aligned}
$$

It is easy now to see that replacing

$$
\begin{aligned}
& G, A, B, C, D, \mu, \nu, L_{\omega} \text { by } \\
& G_{1}, G, H, C, D, \mu_{1}, \nu, M_{\omega} .
\end{aligned}
$$


the relations (1.5)-(1.9) will be preserved. As a matter of fact we have :

(i) for any $\omega, \omega^{\prime} \epsilon \Omega$,

$$
M_{\omega}=\left(L_{\mu \omega} \mu_{1} \cup L_{\omega}\right)^{G_{1}} \subseteq\left(L_{\mu \omega \omega^{\prime}} \mu_{1} \cup L_{\omega \omega^{\prime}}\right)^{G_{1}}=M_{\omega \omega^{\prime}}
$$

since $L_{\mu \omega} \subseteq L_{\omega \omega \omega^{\prime}}$ and $L_{\omega} \subseteq L_{\omega \omega^{\prime}}$, by (1.5).

$$
\begin{aligned}
M_{\mu} \cap G & =L_{\mu}, \quad \text { by }(2.11), \\
& =\text { kernel of } \mu_{1}, \\
M_{\nu} \cap & =L_{\nu} \cap, \quad \text { by }(2.11) \\
& =\text { kernel of } \nu, \\
\left(M_{\mu \omega} \cap G\right) \mu_{1} & =L_{\mu \omega} \mu_{1}, \quad \text { by }(2.11), \\
& =M_{\omega} \cap H, \quad \text { by }(2.12), \\
\left(M_{\nu \omega} \cap C\right) \nu & =\left(L_{\nu \omega} \cap C\right) \nu, \quad \text { by }(2.11), \\
& =L_{\omega} D, \quad \text { by }(1.9), \\
& =M_{\omega} \cap D, \quad \text { by }(2.11) .
\end{aligned}
$$

For the convenience of the notation, we write in $G_{1}: v_{1}=v$. Thus we can repeat the process, embedding $G_{1}$ in $G_{2}$ in which $\nu_{1}$ is extended to $\nu_{2}$ and $\mu_{1}=\mu_{2}$ is unchanged. We carry on inductively, extending $\mu$ and $\nu$ alternatively. Finally we form

$$
G^{*}=\bigcup_{n=1}^{\infty} G_{n}
$$

Define the mappings $\mu^{*}$ and $\nu^{*}$ of $G^{*}$ as follows : For any $g \epsilon G^{*}$, that is to say, $g \epsilon G_{n}$ for some suitable $n$, we.put

$$
g \mu^{*}=g \mu_{n} \quad \text { and } \quad g \nu^{*}=g \nu_{n} .
$$

Thus $\mu^{*}$ and $\nu^{*}$ become total endomorphisms of $G^{*}$ which extend $\mu$ and $\nu$ respectively, and we get the following

\section{Theorem 2.}

The necessary conditions (1.5)-(1.9) of Theorem 1 are also sufficient for $\mu$ and $\nu$ to be totally extendable to two endomorphisms of one and the same group.

By a classical procedure of transfinite induction we can generalize the results of Theorems 1 and 2 , thus obtaining

\section{Theorem 3.}

Let $\mu(\alpha)$, where $\alpha$ ranges over a well-ordered set $\Sigma$ whose ordinal is $\sigma$, be a homomorphism of a subgroup $A_{\alpha}$ of a group $G$ onto a second subgroup $B_{\alpha}$ of $G$. Then the necessary and sufficient conditions for the existence of a group $G^{*} \equiv G$ with endomorphisms $\mu^{*}(\alpha)$ extending $\mu(\alpha)$ is that if we denote by $\Omega$ the semigroup freely generated by the $\mu(\alpha)$, then for every $\omega \epsilon \Omega$ there exists a normal subgroup $L_{\omega}$ of $G$ such that

$$
\begin{aligned}
& L_{\omega} \subseteq L_{\omega \omega_{1}} \text { for all } \omega, \omega_{1} \in \Omega, \\
& L_{\mu(\alpha) \cap} A_{\alpha} \text { is the kernel of } \mu(\alpha), \\
& \left(L_{\mu(\alpha) \omega} \cap A_{\alpha}\right) \mu(\alpha)=L_{\omega} \cap B_{\alpha}, \ldots \ldots
\end{aligned}
$$

for every $\alpha \epsilon \Sigma$ and $\omega \in \Omega$.

The proof is omitted.

\section{§ 3. Some Special Cases :}

In this paragraph some special cases of Theorem 2 are derived ; they are stated for two partial endomorphisms, but they may as well be formulated for any well-ordered set of them. 


\section{Corollary 1.}

For $\mu$ and $\nu$ to be totally extendable to one and the same group, it is sufficient that there exists two non-decreasing sequences

and

$$
\begin{gathered}
L_{1} \subseteq L_{2} \subseteq \cdots \\
M_{1} \subseteq M_{2} \subseteq \cdots
\end{gathered}
$$

of normal subgroups in $G$ such that

$$
\begin{aligned}
& L_{1} \cap A \text { is the kernel of } \mu, \\
& M_{1} \cap C \text { is the kernel of } \nu, \\
&\left(L_{n+1} \cap A\right) \mu=L_{n} \cap B, \\
&\left(M_{n+1} \cap C\right) \nu=M_{n} \cap D, \\
& L_{n} \cap D=\{1\}, \\
& M_{n} \cap B=\{1\},
\end{aligned}
$$

for $n=1,2, \ldots$.

For we can then satisfy conditions (1.5)-(1.9) by putting

$$
\begin{aligned}
& L_{\mu^{n} \omega}=L_{\mu^{n}}=L_{n} \quad \text { for } \omega=\nu \omega^{\prime}, \\
& L_{\nu n_{\omega}}=L_{\nu n}=M_{n} \text { for } \omega=\mu \omega^{\prime},
\end{aligned}
$$

for all $n=1,2, \ldots$.

Corollary 2.

Sufficient for $\mu$ and $\nu$ to be totally extendable to one and the same group that if $K$ is the kernel of $\mu$ and $K^{\prime}$ is the kernel of $\nu$, then

$$
\begin{aligned}
& K^{Q} \cap A=K \text {, } \\
& K^{\prime G} \cap C=K^{\prime} \\
& K^{G}{ }_{n} B=K^{G}{ }_{n} D=K^{\prime G}{ }_{n} B=K^{\prime G}{ }_{n} D=\{1\} \text {. }
\end{aligned}
$$

For then we satisfy the conditions of the theorem by putting

for all $\omega \epsilon \Omega$.

$$
\begin{aligned}
& L_{\mu \omega}=L_{\mu}=K^{\theta}, \\
& L_{\nu \omega}=L_{\nu}=K^{\prime} \theta
\end{aligned}
$$

We note that in the corresponding corollary by B. H. Neumann and Hanna Neumann (2, Corollary 6.3), it is not sufficient (as there stated) to have $K^{a} \cap B=\{1\}$; for we must have relation (3.1) together with it.

Before formulating the third corollary, we give a name for a certain kind of subgroup $(1$, Theorem V) and $(2, \S 6)$ :

Definition.

The subgroup $S$ of the group $R$ is called an " $E$-subgroup " of $R$ if every normal subgroup of $S$ is the intersection with $S$ of a normal subgroup of $R$; or equivalently if, for every normal subgroup $T$ of $S$,

\section{Corollary 3 :}

$$
T^{R} \cap S=T
$$

Sufficient for $\mu$ and $\nu$ to be totally extendable to one and the same group that $A$ and $C$ are $E$-subgroups of $G$.

Proof :

We define $K_{\mu}=1 \mu^{-1}$, and inductively

$$
K_{\mu \omega}=K_{\omega}^{A} \mu^{-1} \text {, }
$$

that is, the greatest subgroup of $A$ mapped into $K_{\omega}^{G}$ by $\mu$. 
Similarly we define $K_{\nu}=1 \nu^{-1}$, and inductively

for all $\omega \epsilon \Omega$. Then we put

$$
K_{\nu \omega}=K_{\omega}^{G} \nu^{-1}
$$

$$
L_{\omega}=K_{\omega}^{G}
$$

To confirm that conditions (1.5)-(1.9) are satisfied, we first prove that for any $\omega, \omega^{\prime} \in \Omega$,

$$
K_{\omega} \subseteq K_{\omega \omega \omega} \text {. }
$$

The proof is by induction on the length $n$ of the word $\omega$.

If $n=1$, that is, if $\omega=\mu$ say, then

$$
x \in K_{\mu} \text { implies } x \mu=1 \in K_{\omega^{\prime}}^{G} \text { for any } \omega^{\prime} \text {. }
$$

Thus $x \in K_{\omega^{\prime}}^{a} \mu^{-1}=K_{\mu \omega^{\prime}}$, and we get

Similarly,

$$
\begin{aligned}
& K_{\mu} \subseteq K_{\mu \omega} \cdot \\
& K_{\nu} \subseteq K_{\nu \omega^{*}}
\end{aligned}
$$

Suppose that (3.4) is true for $\omega$, then

$$
x \in K_{\mu \omega} \text { implies } x \mu \in K_{\omega}^{G} \subseteq K_{\omega \omega}^{G} .
$$

Thus $x \in K_{\omega \omega,}^{G} \mu^{-1}=K_{\mu \omega \omega^{\prime}}$ and we get

Similarly

$$
\begin{aligned}
K_{\mu \nu} & \subseteq K_{\mu \omega \omega^{\prime}} \\
K_{\nu \omega} & \subseteq K_{\nu \omega \omega^{\prime}} .
\end{aligned}
$$

This completes the proof of (3.4), from which it follows that

and this confirms (1.5).

$$
L_{\omega} \subseteq L_{\omega \omega^{\prime}} \text {, }
$$

Since $A$ and $C$ are $E$-subgroups of $G$ and $K_{\mu}$ is normal in $A, K_{\nu}$ is normal in $C$, then

$$
\begin{aligned}
& K_{\mu}^{G} \cap A=K_{\mu}, \\
& K_{\nu}^{G} \cap C=K_{\nu},
\end{aligned}
$$

and thus

$$
\begin{aligned}
& L_{\mu} \cap A \text { is the kernel of } \mu, \\
& L_{\nu} \cap C \text { is the kernel of } \nu,
\end{aligned}
$$

which confirm (1.6) and (1.7).

To confirm (1.8) and we note that

$$
L_{\mu \omega} \cap A=K_{\mu \omega}^{G} \cap A,
$$

and since $K_{\mu \omega}$ is normal in $A$, and $A$ is an $E$-subgroup of $G$, then

$$
L_{\mu \omega} \cap A=K_{\mu \omega} \text {. }
$$

By the definition of $K_{\mu \omega}$ we have

Since $K_{\mu \omega} \mu \subseteq B$, then

$$
K_{\mu \omega} \mu \subseteq K_{\omega}^{G}
$$

$$
K_{\mu \omega} \mu \subset K_{\omega}^{G} \cap B .
$$

If, on the other hand, $b \in K_{\omega}^{G} \cap B$, then there exists an element $a \in A$ such that

thus

$$
b=a \mu \in K_{\omega}^{G}
$$$$
a \in K_{\omega}^{G} \mu^{-1}=K_{\mu \omega} \text {, }
$$

and thus

$$
\begin{gathered}
b=a \mu \in K_{\mu \omega} \mu, \\
K_{\omega}^{G} \cap B \subseteq K_{\mu \omega} \mu .
\end{gathered}
$$


(3.6) and (3.7) together give

Or using (3.5),

$$
K_{\mu \omega} \mu=K_{\omega}^{G} B .
$$

Similarly one shows that

$$
\left(L_{\mu \omega} \cap A\right) \mu=L_{\omega} \cap B \text {. }
$$

$$
\left(L_{\nu \omega} \cap C\right) \nu=L_{\omega} \cap D .
$$

This completes the proof of Corollary 3 .

Again, we note that in the corresponding lemma by B. H. Neumann and Hanna Neumann (2, Corollary 6.4), one has to define $K=K_{1}=1 \mu^{-1}$ and $K_{n+1}$ inductively as

$$
K_{n+1}=K_{n}^{G} \mu^{-1},
$$

and not (as in the paper referred to) as $K_{n+1}=K_{n} \mu^{-1}$.

A very special case of Corollary 3 is the following :

Corollary 4.

$\mu$ and $\nu$ are always extendable to one and the same group if $G$ is abelian. For in an abelian group every subgroup is an $E$-group.

But as a matter of fact, Theorem 4 says more than that, namely:

\section{Theorem 4.}

$\mu$ and $\nu$ are always extendable to one and the same abelian group if $G$ is abelian. Proof.

Let $K$ and $K^{\prime}$ be the kernels of $\mu$ and $\nu$ respectively, and let $\mu_{1}$ be the canonic mapping of $G$ onto $G / K=H$. Form the direct product $G_{1}$ of $G$ and $H$ amalgamating $B$ with $A / K$ according to $\mu_{1}$ :

$$
G_{1}=\{G \times H ; \quad B=A / K\} .
$$

$G_{1}$ is abelian and contains the subgroup $G$ mapped by $\mu_{1}$ onto $H$ and the subgroup $C$ mapped by $\nu_{1}=\nu$ onto $D$.

Repeat the process, embedding $G_{1}$ in $G_{2}$, extending $\nu_{1}$ to $\nu_{2}$ and leaving $\mu_{1}=\mu_{2}$ as it is. We continue indefinitely, then form

$$
G^{*}=\bigcup_{n=1}^{\infty} G_{n},
$$

which is evidently abelian and if we define $\mu^{*}$ and $\nu^{*}$ as follows : for any $g \epsilon G^{*}$, that is to say, $g \in G_{n}$ for some suitable $n$ we put $g \mu^{*}=g \mu_{n}$ and $g \nu^{*}=g \nu_{n}$, then $G^{*}$ satisfies the required conditions.

We conclude by stating without proof the following

\section{Theorem 5:}

Necessary and sufficient conditions for the partial endomorphism $\mu$ which maps $A$ onto $B$ and the partial endomorphism $v$ which maps $C$ onto $D ; A, B, C, D$ being subgroups of a given group $G$, to be totally extendable to one and the same total endomorphism of a group $G^{*} \equiv G$ is that if we define $\pi$ to map any word $w(a, c) \in\{A, C\}$ onto $w(a \mu, c \nu) \in\{B, D\}$ where $a \in A, c \in C$, then

$\pi$ is a one-valued mapping of $\{A, C\}$ onto $\{B, D\}$ which is a homomorphism,

there exists a non-decreasing sequence

of normal subgroups in $G$ such that

$$
L_{1} \text { 드 } L_{2} \subseteq \ldots
$$

$$
\begin{aligned}
& L_{1} \cap\{A, C\} \text { is the kernel of } \pi, \\
& \left(L_{n+1} \cap\{A, C\}\right) \pi=L_{n} \cap\{B, D\},
\end{aligned}
$$

for all $n=1,2, \ldots$. 
(1) Higman, G., Neumann, B. H., and Neumann, H., "Embedding theorems for groups," J. London Math. Soc., 24 (1949), 247-254.

(2) Neumann, B. H., and Neumann, Hanna, " Extending partial endomorphisms of groups," Proc. London Math. Soc., (3) 2, (1952), 337-348.

Department of Mathematics

The UnIVERSITY

MANChester 13

and

Faculty of Science

The UnIVersity

Alexandria 